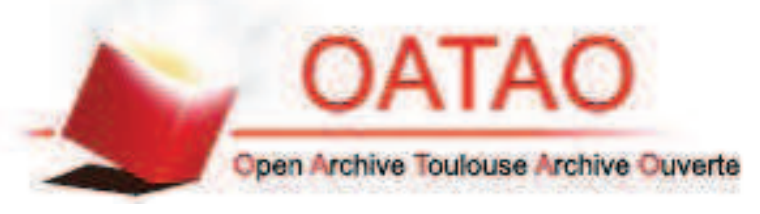

OATAO is an open access repository that collects the work of Toulouse researchers and makes it freely available over the web where possible.

This is an author-deposited version published in : http://oatao.univ-toulouse.fr/ Eprints ID : 10533

To link to this article : DOI: $10.1209 / 0295-5075 / 98 / 34003$

http://dx.doi.org/10. 1209/0295-5075/98/34003

To cite this version : Oh, J. M. and Legendre, Dominique and Mugele, Frieder Shaken not stirred -On internal flow patterns in oscillating sessile drops. (2012) Europhysics Letters, vol. 98 (n³). ISSN 0295-5075

Any correspondance concerning this service should be sent to the repository administrator: staff-oatao@listes-diff.inp-toulouse.fr 


\title{
Shaken not stirred -On internal flow patterns in oscillating sessile drops
}

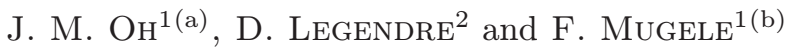 \\ ${ }^{1}$ Physics of Complex Fluids Group, TNW, University of Twente - NL-7500AE, Enschede, The Netherlands, EU \\ ${ }^{2}$ Université de Toulouse, INPT, UPS, Institut de Mécanique des Fluides de Toulouse (IMFT) \\ Allée Camille Soula, F-31400 Toulouse, France, EU
}

\begin{abstract}
We use numerical (volume of fluid) simulations to study the flow in an oscillating sessile drop immersed in an ambient immiscible fluid. The drop is excited by a sinusoidal variation of the contact angle at variable frequency. We identify the eigenfrequencies and eigenmodes of the drops and analyze the internal flow fields by following the trajectories of tracer particles. The flow fields display an oscillatory component as well as a time-averaged mean component. The latter is oriented upward along the surface of the drop from the contact line towards the apex and downward along the symmetry axis. It vanishes at high and low frequencies and displays a broad maximum around $f=200-300 \mathrm{~Hz}$. We show that the frequency dependence of the mean flow can be described in terms of Stokes drift driven by capillary waves that originate from the contact line, in agreement with recent experiments (MugELE F. et al., Lab Chip, 11 (2011) 2011).
\end{abstract}

Introduction. - The dynamics of oscillating sessile drops are of great interest to many fundamental and practical problems including the impact of drops on solid surfaces, the generation of drops (e.g. in inkjet printers), and the manipulation of drops in microfluidic and optofluidic applications. Compared to the classical fluid dynamics problem of oscillating free drops, for which Rayleigh [1] developed the first systematic solution, the problem of sessile drops is more complex because the presence of the solid surface reduces the symmetry and gives rise to additional dissipation due to the adhesion of the liquid to the solid surface $[2,3]$. Moreover, it introduces a contact line that can be either pinned or mobile and a contact angle with a velocity-dependent dynamic value. As a consequence, the absolute values of the eigenfrequencies of the drops shift while their scaling with surface tension, density, and drop size remains unaffected [4]. Novel applications inspired by microfluidics trigger questions beyond the previous focus on eigenfrequencies and eigenmodes including the coupling between drop resonances, contact angle hysteresis and drop motion [4-6] as well as timeaveraged streaming flows that promote mixing within the

\footnotetext{
(a) E-mail: jmo0902@gmail.com

(b) E-mail: f.mugele@utwente.nl
}

drops [7-10] and affect the deposition of residues from evaporating drops [11]. In those experiments drop oscillations are typically excited either by mechanical shaking or by a periodic modulation of the equilibrium contact angle using electrowetting (EW).

The goal of the present numerical study is to characterize the flow patterns in oscillating drops and to elucidate their physical origin. Inspired by EW experiments [7-11], we adopt a numerical scheme based on the volume of fluid (VOF) method with a diffuse interface that is able to deal with both moving contact lines and viscous ambient fluids [12]. This approach extends the scope of earlier numerical studies $[3,13]$ that focused on shape oscillations, eigenfrequencies, and instantaneous flow fields in the numerically simpler situation of drops with pinned contact lines in ambient air. We analyze the drop oscillations in terms of eigenmodes and characterize instantaneous and mean flows by following the trajectories of passive tracer particles. Despite the presence of pronounced resonances the simulations are found to reproduce all important characteristics including the frequency dependence of the mean flow reported in the experiments. The origin of the mean flow can be described consistently with a Stokes drift model that is based on propagating capillary waves rather than discrete eigenmodes [9]. 


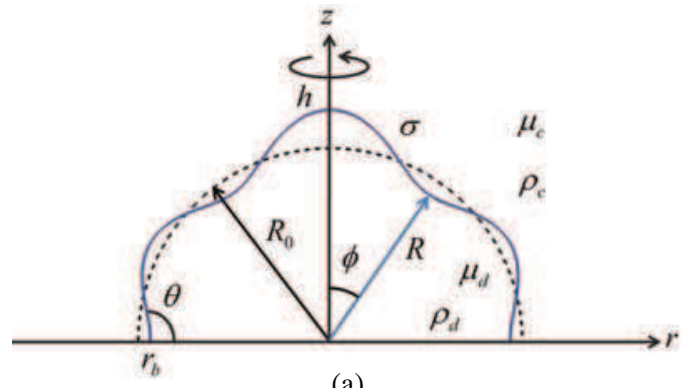

(a)

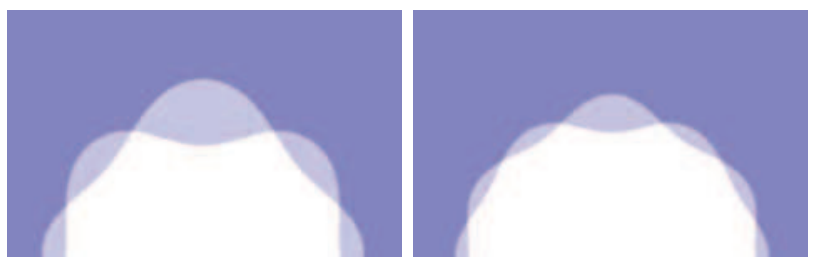

(b)

Fig. 1: (Color online) (a) System description of a drop in viscous medium on a wetted substrate and (b) oscillation patterns at $176 \mathrm{~Hz}$ (left) and $314.6 \mathrm{~Hz}$ (right).

Problem definition and numerical implementation. - We consider a sessile drop placed on a flat substrate in an immiscible viscous liquid bath as shown in fig. 1(a). The physical parameters are chosen to mimic typical experiments with millimeter-sized aqueous drops in ambient oil [9]: The equilibrium shape of the drop is a hemisphere with Young's angle $\theta_{Y}=90^{\circ}$ and with an average radius $R_{0}=1 \mathrm{~mm}$. The densities and viscosities of drop and surrounding continuous medium are $\rho_{d}=\rho_{c}=$ $1000 \mathrm{~kg} / \mathrm{m}^{3}, \mu_{d}=1 \mathrm{cP}$ and $\mu_{c}=6 \mathrm{cP}$, respectively. The surface tension between the two fluids is $\sigma=35 \mathrm{mN} / \mathrm{m}$. Drop oscillations are induced by time-periodic variations of the equilibrium contact angle $\theta(t)$ on the substrate following

$$
\cos \theta(t)=\cos \theta_{0}+\Delta \sin (2 \pi f t) .
$$

Here $f$ and $\Delta$ are the forcing frequency and amplitude, respectively. There is no contact angle hysteresis. Experimentally, such contact angle variations are easily realized over a wide range of forcing frequencies using electrowetting (EW) [7-9].

We solve the full Navier-Stokes equations and continuity equation with a surface tension force on the free interface in a single axisymmetric domain. The numerical scheme is implemented using the volume of fluid (VOF) method with a continuous surface force formalism (CSF). The calculations are carried out in a domain of interest of $3 \mathrm{~mm} \times 3 \mathrm{~mm}$ with a $300 \times 300$ staggered grid system in the cylindrical coordinates $(r, z)$. For more details of the method, implementation, and validation we refer the reader to ref. [12], which also describes the optimized regularization and filtering schemes used in the present work. Unless stated otherwise, the forcing amplitude is $\Delta=1 / 2$, corresponding to $60^{\circ}<\theta(t)<120^{\circ}$, and the

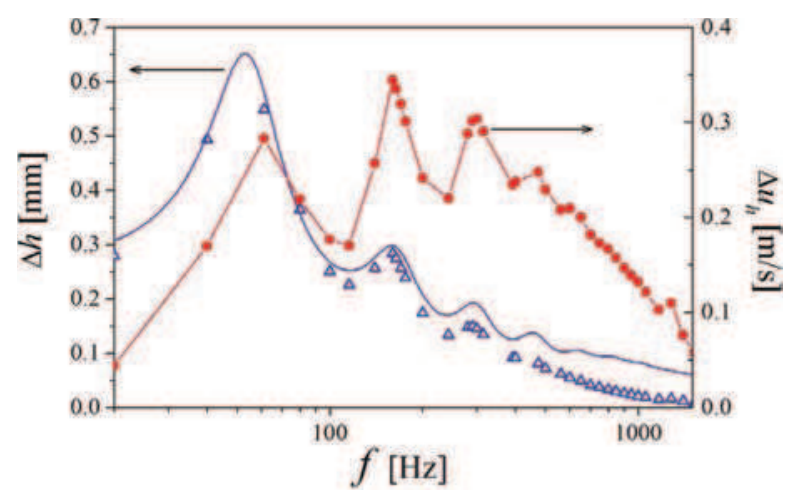

Fig. 2: (Color online) Frequency dependence of oscillation amplitudes of height ( $\Delta h$, blue triangles) of drop, the corresponding speeds $\left(\Delta u_{h}\right.$, red circles) and reconstruction of $\Delta h$ based on harmonic oscillator model (blue solid line).

forcing frequencies are in the range of $20 \mathrm{~Hz}<f<2000 \mathrm{~Hz}$. Since we are interested in steady-state oscillations we run the simulations for at least 100 oscillations cycles for each condition with at least 2000 time steps per period. We compare the flow fields at the beginning and the end of these two last cycles to verify that transients have decayed. All results discussed below are extracted from the last two oscillation cycles.

Drop oscillations. - The response of the liquid surface depends strongly on the forcing frequency. At low frequencies $(f<600 \mathrm{~Hz})$, a series of resonances at distinct eigenfrequencies are observed as illustrated by the snapshots in fig. 1(b). At higher forcing frequencies the amplitude of the surface progressively decreases. Figure 2 illustrates this behavior using the amplitude $\Delta h$ of the apex of the surface at $r=0$ as well as the corresponding speed $\Delta u_{h}$. The latter is found to agree with $\mathrm{d} \Delta h / \mathrm{d} t=2 \pi f \Delta h$ within the numerical accuracy. $\Delta h$ vanishes at high frequencies as expected. For $f \rightarrow 0, \Delta h$ approaches a constant value $\Delta h_{\min }=0.262 \mathrm{~mm}$ as determined by the drop size and the forcing amplitude. $\Delta u_{h}$ vanishes both at low and at high frequencies. In between, it displays a broad maximum around $f \approx 200 \mathrm{~Hz}$ with superimposed local maxima due to the resonances. While the behavior at low frequencies is a trivial consequence of the saturation of $\Delta h$, the high frequency limit implies that $\Delta h$ vanishes faster than $1 / f$ for high frequencies.

To analyze the drop oscillations we expand the surface shapes into the Legendre polynomials, i.e. we write the surface profile $R(\phi, t)$ as

$$
R(\phi, t)=R_{0}+\sum_{n} a_{n}(t) P_{n}(\cos \phi),
$$

where $P_{n}$ is the Legendre polynomial of order $n$. Since the Legendre polynomials form a complete and orthogonal basis in $-1 \leqslant \cos \phi \leqslant 1$, we can calculate the coefficients $a_{n}$ of the shape modes from the numerically obtained surface 


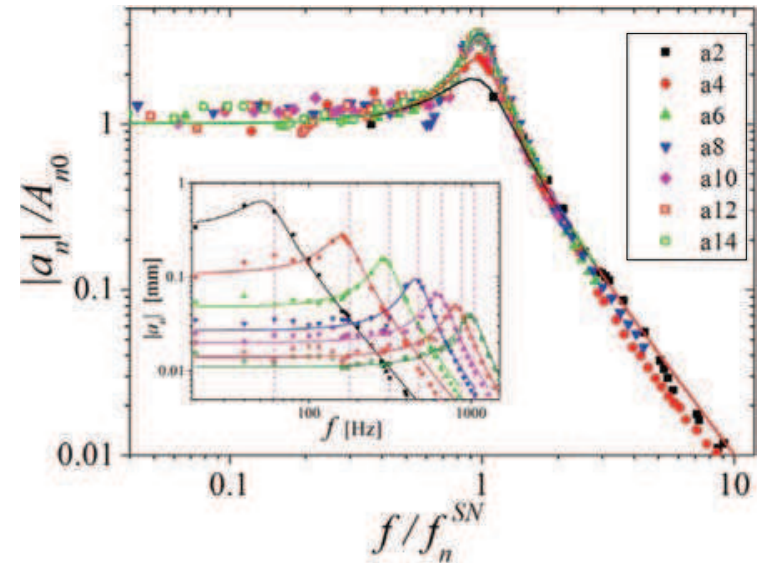

Fig. 3: (Color online) Frequency dependence of the amplitudes of shape modes. Symbols denote numerical results and solid lines represent harmonic oscillator fit curves. The vertical red dashed lines in the inset represent the resonance frequencies predicted by eq. (4).

profile $R(\phi, t)$ as

$$
a_{n}(t)=\frac{2 n+1}{2} \int_{-1}^{1} R(\cos \phi, t) P_{n}(\cos \phi) \mathrm{d}(\cos \phi) .
$$

For symmetry reasons, this expansion contains only even modes for the present conditions [14]. (This is readily confirmed by comparing $R(\phi, t)$ to the reconstructed shape based on the $a_{n}$.) The symbols in the inset of fig. 3 show the absolute values of the resulting coefficients $\left|a_{n}\right|$ for $n=2,4,6, \ldots, 14$ as obtained from the numerical surface profiles. Each individual mode follows largely the response of a damped harmonic oscillator with a specific resonance frequency and damping coefficient (see solid lines in fig. 3). The resonance frequencies $f_{n}^{S N}$ extracted from the fits are shown in table 1. Except for a small variation of the damping, the amplitude coefficients collapse onto the universal response function of a damped harmonic oscillator if the amplitudes and the frequency are normalized by their low frequency limits and by the resonance frequencies $f_{n}^{S N}$, respectively (see main panel of fig. 3). Notwithstanding the presence of some non-linear coupling for the lowest eigenmodes (in particular between the $P_{2}$ and the $P_{4}$ mode) and a slight systematic over estimation of the $a_{n}$ at high frequencies ${ }^{1}$, this linear analysis overall provides a decent description of the drop dynamics. This is also consistent with the reconstruction of $\Delta h$ based on the harmonic oscillator model (blue solid line in fig. 2). It implies in particular that the amplitudes $a_{n}$ of the eigenmodes vanishes as $\propto 1 / f^{2}$ for $f \gg f_{n}^{*}$ and thereby explains the vanishing of $\Delta u_{h}$ in fig. 2 at high frequencies.

The vertical dashed lines in the inset of fig. 3 denote the resonance frequencies for a free drop in a viscous medium

\footnotetext{
${ }^{1}$ At high frequencies, the $a_{n}$ 's become smaller than the grid size and thus less reliable (see inset of fig. 3).
}

Table 1: Comparison of resonance frequencies.

\begin{tabular}{crrr}
\hline$n$ & \multicolumn{1}{c}{$f_{n}^{*}$} & \multicolumn{1}{c}{$f_{n}^{t h}$} & \multicolumn{1}{c}{$f_{n}^{S N}$} \\
\hline 2 & 65.23 & 61.19 & 55.02 \\
4 & 188.32 & 175.95 & 164.14 \\
6 & 338.48 & 314.53 & 300.23 \\
8 & 512.68 & 474.13 & 464.73 \\
10 & 708.20 & 652.22 & 640.86 \\
12 & 923.01 & 846.94 & 824.10 \\
14 & 1155.57 & 1056.83 & 1016.04 \\
\hline
\end{tabular}

following the classical theory by Miller and Scriven [15]. The latter are given by

$$
f_{n}^{t h}=f_{n}^{*}-\frac{\mu_{d}}{\rho_{d} R_{0}^{2}} F \sqrt{R e_{d, n}}
$$

where $f_{n}^{*}$ is the resonance frequency for potential flow

$$
f_{n}^{*}=\frac{1}{2 \pi} \sqrt{\frac{(n-1) n(n+1)(n+2) \sigma}{\left(\rho_{d}(n+1)+\rho_{c} n\right) R_{0}^{3}}} .
$$

$F$ is the viscous correction term

$$
F=\frac{(2 n+1)^{2}(\hat{\rho} \hat{\mu})^{\frac{1}{2}}}{4 \sqrt{2} \pi[n \hat{\rho}+n+1]\left[1+(\hat{\rho} \hat{\mu})^{\frac{1}{2}}\right]},
$$

where the density ratio $\hat{\rho}$ and viscosity ratio $\hat{\mu}$ are given as $\hat{\rho}=\rho_{c} / \rho_{d}$ and $\hat{\mu}=\mu_{c} / \mu_{d}$, respectively.

Overall, the individual resonance frequencies are rather close to the values obtained for free drops. This result is not too surprising since the surface tension and the mass involved in the oscillations are the same as in the case of free drops. The additional viscous damping due to the presence of the moving contact line as well as the viscous boundary layer at the drop-substrate interface produces only a rather small relative shift, as indicated by the similarity of the values of $f_{n}, f_{n}^{*}$, and $f_{n}^{S N}$ in table 1 . (Note that the apparently larger relative shift of the $f_{2}$ mode may be affected by non-linear coupling [16].) The rather small viscosity-induced shifts are consistent with the overall Reynolds number

$$
R e_{d, n}=\frac{\rho_{d} R_{0}^{2}\left(2 \pi f_{n}^{*}\right)}{\mu_{d}}
$$

which ranges from 410 to 14048 in $20 \mathrm{~Hz}<f<2048 \mathrm{~Hz}$ indicating inertia-dominated dynamics.

Close inspection of the video sequences from the simulations reveals another important characteristic of the drop response. Due to the specific forcing mechanism of imposing a time-dependent contact angle, the "information" to change the shape enters the drop from the contact line and subsequently spreads further. At high forcing frequencies, this generates very clearly the impression of propagating waves traveling from the contact line towards the apex of 


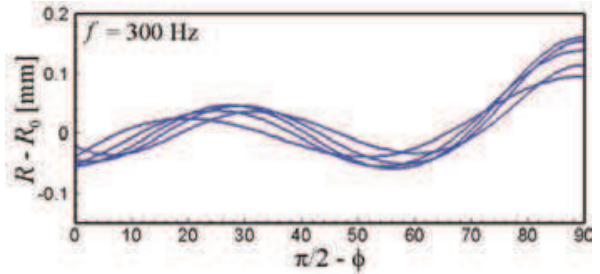

(a)

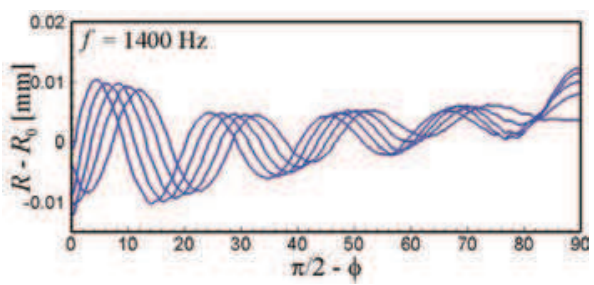

(b)

Fig. 4: (Color online) Wave propagation on the drop surface from the contact line to the apex of the drop at (a) $300 \mathrm{~Hz}$ and (b) $1400 \mathrm{~Hz}$. Each curve is plotted at every (a) $0.08 \mathrm{~T}$ (2.667e$4 \mathrm{~s})$ and (b) $0.1 T(7.143 \mathrm{e}-5 \mathrm{~s})$, respectively.

the drop. Figure 4(b) shows this for $f=1400 \mathrm{~Hz}$. Note also that the wave is slightly damped. As a consequence, the reflected wave propagating back from the apex towards the contact line has a smaller amplitude than the original one causing the appearance of a travelling wave. (The increase for $\phi \rightarrow 0$ in fig. 4(b) is due to a geometric focusing effect at $r=0$.) At lower frequencies, in particular close to the resonance frequencies, the drop shapes appear more like standing wave patterns. Yet, also under these conditions a gradual propagation of nodes and antinodes from the substrate towards the drop apex can be observed (see fig. 4(a)). Interestingly, the propagation speed of these waves turns out to be approximately constant and assumes a value of $\approx 0.4 \mathrm{~m} / \mathrm{s}( \pm 10 \%)$ for the conditions studied here. The propagation speed agrees fairly well with the phase velocity for capillary wave $\left(v_{p}=f \lambda \sim 0.4-0.5 \mathrm{~m} / \mathrm{s}\right)$ where $\lambda$ is the wavelength.

Internal flow. - We now turn to the flow fields within the drop. Since the shape oscillations are perfectly periodic in time, the local flow field at any fixed position $\mathbf{x}_{0}$ in space both within and outside the drop is also periodic in time. For mixing or other problems involving mass transfer within the drop, however, it is more interesting to consider the trajectory of volume elements of fluid or of passive tracer particles following the flow. Lagrangian particle tracing leads to trajectories

$$
\mathbf{x}=\mathbf{x}_{0}+\int_{t_{0}}^{t} \mathbf{u}(\mathbf{x}, t) \mathrm{d} t
$$

that are not necessarily periodic in time. Here $\mathbf{u}(\mathbf{x}, t)$ is the velocity of the fluid element at position $\mathbf{x}$ and time $t$. Figure 5(a) shows the motion of a many tracer particles followed of a duration of $\approx 9$ cycles. In contrast to the drop surface, none of the tracer trajectories is periodic in

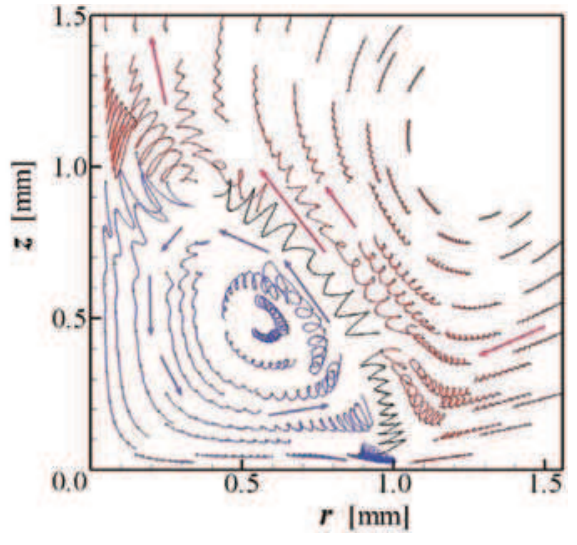

(a)

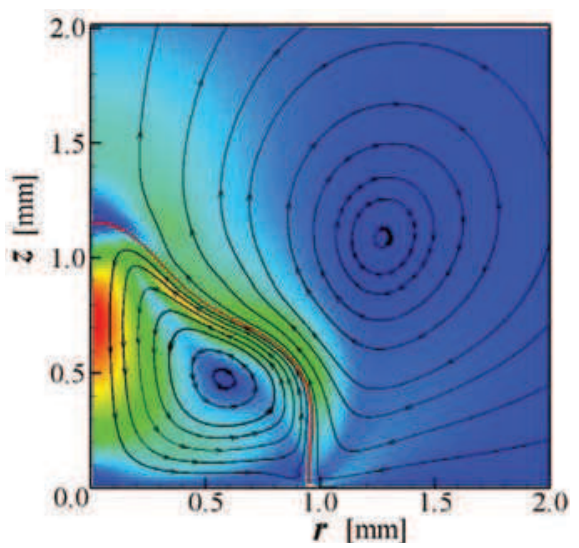

(b)

Fig. 5: (Color online) (a) Particle trajectories inside (blue) and outside (red) drop at $f=300 \mathrm{~Hz}$. Arrows: direction of mean flow. (b) The corresponding net flow where color contour represents absolute net speed $u=|\mathbf{u}|$ (color scale: $u_{\max }=$ $0.055 \mathrm{~m} / \mathrm{s}$ (red); $u=0$ (blue)).

time. Rather, all particles display a net displacement per oscillation cycle superimposed on an oscillatory motion. Small dots indicate the positions of the particles once per oscillation cycle. Both the amplitude of the oscillatory motion as well as the strength of the net drift motion depend on the position. Particularly vigorous motion is observed close to the contact line and close to the apex of the drop, where the amplitude is particularly pronounced due to geometric focusing. The net motion with color contour shown in fig. 5(b) is directed upward from the contact line towards the apex of the drop. Within the drop this leads to a vortex rotating counterclockwise with a maximum flow velocity along the the symmetry axis of the drop in the downward direction. In the ambient phase, a second "mirror" vortex develops, which also rotates clockwise to satisfy continuity of shear stress at the drop surface. Particles on the interface display a zigzag motion. Qualitatively similar patterns are observed at other forcing frequencies up to approximately $1 \mathrm{kHz}$ both for the instantaneous and for the mean flow. At higher frequencies additional more complex flow patterns arise 
close to the apex of the drop. Given their overall weakness we disregard these flows in the remainder of this work.

To characterize the overall strength of the mean flow as a function of the frequency we calculate its volume average from the time-averaged motion of $N \approx 14000$ tracer particles uniformly distributed within the drop according to

$$
\langle u(f)\rangle=\frac{\int_{\Omega} u \mathrm{~d} V}{\int_{\Omega} \mathrm{d} V} \simeq \sum_{i}^{N} r_{i} u_{i} / \sum_{i}^{N} r_{i}
$$

Here $u=|\mathbf{u}|$ and $r_{i}$ is the distance of the $i$-th particle from the symmetry axis. The result, shown as red solid circles in fig. 7 , displays a broad maximum around $f \approx 200 \mathrm{~Hz}$ with a series of superimposed maxima at frequencies close to the eigenfrequencies of the drop. For both low and high frequencies $\langle u\rangle$ vanishes. Overall, the frequency dependence of the mean flow is thus very similar as the velocity $\Delta u_{h}$ at the apex of the drop.

Stokes drift. - How can we understand the physical origin and the frequency dependence of the mean flow? Qualitatively, it is natural that the mean flow ceases for low frequencies. In that limit, the spreading and receding motion of the drop is quasi-static and hence perfectly reversible. It is also plausible that the mean flow vanishes at very high frequencies. In that limit inertia prevents the drop from following the excitation and the drop shape becomes quasi-stationary. In discussing fig. 4 we noted that perturbations of the surface travel in a wave-like fashion from the contact line towards the apex of the drop. At the surface, the mean flow in our simulations propagates in the same direction as these capillary waves. In our previous experimental work [9] we observed similar patterns and suggested that the flow can be explained in terms of "Stokes drift". The present numerical calculations allow for a more detailed comparison to this model. In general, Stokes drift describes the net transport of tracer particles along the direction of propagation of waves at liquid interfaces. The origin of this effect can be understood as follows: tracer particles inside the fluid follow almost circular trajectories. The diameter of these trajectories is maximum at the surface and decreases exponentially with increasing distance from the interface with a decay length of order of the wavelength $\lambda$ (see fig. 6). To first order, the displacement velocity at any given moment $t$ is exactly compensated by the displacement velocity at $t+T / 2$, where $T$ is the period of the wave. Then, the trajectories will make a closed circle to the first order (see the lhs in fig. 6). Yet, since the velocity is $z$-dependent, the horizontal velocity component at the lowest point of the trajectory is somewhat smaller than the one at the highest point of the trajectory. Therefore, for finite drive amplitudes the two components do not perfectly compensate but give rise to a net horizontal displacement, the Stokes drift (see the rhs of fig. 6). The

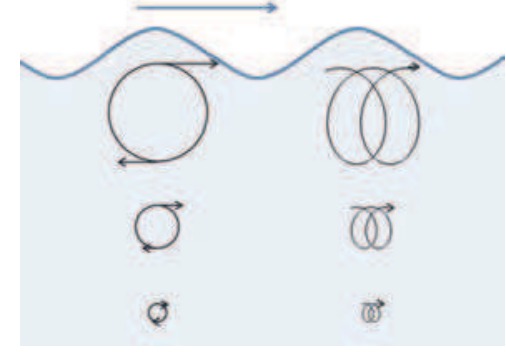

Fig. 6: (Color online) Schematic particle trajectories below a surface with a travelling wave. Left: linearized situation with circular trajectories. Right: second-order trajectories displaying Stokes drift (see text for details)

drift velocity is given by

$$
\langle u(f, z)\rangle \propto f k A(f)^{2} e^{-2 k z} \propto v_{p}\left[\frac{A(f)}{\lambda}\right]^{2} e^{-2 k z} .
$$

Here, $A$, and $k=2 \pi / \lambda$ are the amplitude, and wave number of the surface wave, respectively. As apparent from the formula, the effect is purely inviscid ${ }^{2}$ and of second order in the small parameter $A / \lambda$.

Adapting this mechanism to our present simulations, we interpret the mean flow as being driven by the capillary waves that travel upward along the drop surface. Indeed the trajectories of tracer particles close to the drop surface resemble the ones in standard Stokes drift, in particular for polar angles of $\phi \approx 45^{\circ}$ where neither the solid substrate nor the focusing of the waves close to the drop apex disturb the flow (see fig. 5(a)). Obviously, the actual flow field within the drop is more complex than in the case of a propagating plane wave on a flat surface. In particular, the finite size of the drop forces the stream lines to close upon themselves and produce the vortex in which the liquid circulates back downward towards the substrate along the symmetry axis. Notwithstanding these differences, the Stokes drift model makes a clear prediction regarding the frequency dependence of the mean flow. Averaging over both time and volume, we expect

$$
\langle u(f)\rangle \propto f^{2} A(f)^{2} / v_{p} \propto f^{2}(\Delta h(f))^{2} .
$$

Here we make use of the facts that $v_{p}=$ const and $\Delta h(f) \propto$ $A(f)$. The mean flow obtained from eq. (11) is shown as blue circles in fig. 7. Up to a single scaling factor to match the absolute value, the data agree remarkably well with the red circles obtained averaging by directly the numerical flow speeds. Equation (11) implies $\langle u(f)\rangle \propto\left(\Delta u_{h}\right)^{2}$, which explains the qualitatively similar behavior as $\Delta u_{h}$ in fig. 2 . To test the dependence of the Stokes drift on $A$ we varied the forcing amplitude of the contact angle variation at a fixed frequency. The inset of fig. 7 shows that $\langle u\rangle$ is indeed quadratic in $A$ as predicted. Thus, we conclude that the

${ }^{2}$ Viscous dissipation gives rise to corrections to eq. (10) due to a viscous boundary layer at the interface, as described recently by Belonozhko and Kozin [17]. 


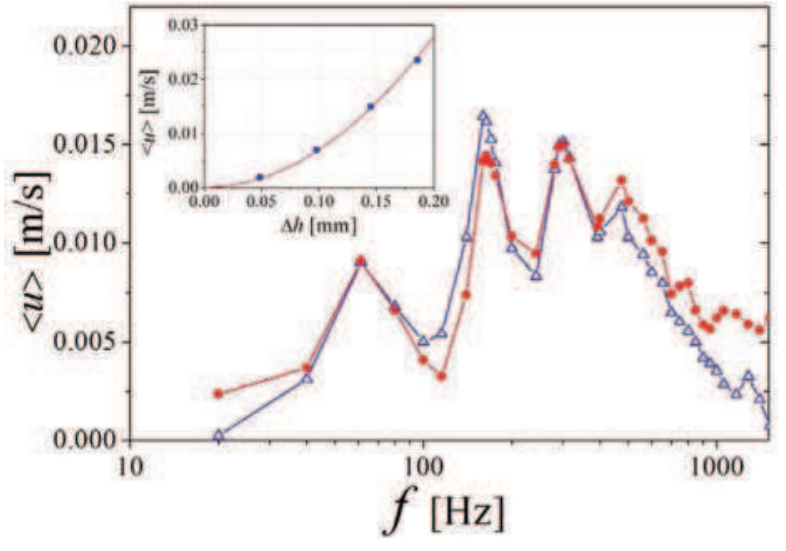

Fig. 7: (Color online) Frequency dependence of the average flow speed. Red line: volume average obtained from numerically simulated flow speed. Blue line: prediction of the Stokes drift model (eq. (11)) using numerical result for $\Delta h$ from fig 2 . Inset: $\langle u\rangle$ vs. $\Delta h$ at $f=300 \mathrm{~Hz}$.

Stokes drift model provides a consistent description of the net flow inside the drop under the conditions studied here.

Discussion. - Comparing the results of fig. 7 to earlier quantitative measurements of the flow fields in oscillating drops $[7,9]$, we find an overall qualitative agreement regarding the broad maximum of the mean flow, the orientation of the flow, as well as the decay at both low and high frequencies. Moreover, we find that tracer particles initially concentrated in the top half of the drop are distributed across the drop within a few hundred oscillation cycles in a very similar manner as the patches of fluorescent dye in refs. [7,9] (see also supplementary movie Suppl-1-mixing.mov). Quantitatively, however, there are deviations from the experiments. The absolute value of the maximum flow speed is substantially higher than in the experiments. Moreover, the simulations display strong resonances which were completely smeared out in the experiments. However, it was noted in ref. [9] that the contact line did not move smoothly in these experiments but displayed temporal pinning and depinning in each oscillation cycle. Such an irregular motion of the contact line leads to a highly non-sinusoidal forcing effectively exciting the drop with many frequencies at the same time. Sudden depinning events probably also give rise to additional damping that can further broaden the resonances. Further studies will be needed to verify these dramatic consequences of a hysteretic contact line motion for the oscillation dynamics of drops.

Conclusion. - In conclusion, the present numerical simulations provide a consistent picture of the oscillation dynamics of sessile drops with moving contact lines in a dense and viscous ambient fluid. The eigenmodes can be described by Legendre polymials and the resonance frequencies are shifted by less than $10 \%$ as compared to free drops. The specific excitation with a modulation of the contact angle gives rise to wave-like perturbations traveling from the contact line towards the apex of the drop. The resulting capillary waves drive a net internal flow that scales with the square of characteristic speed of the oscillating drop surface and thus vanishes for both low and high frequencies. The present calculations provide a starting point for a number of open problems to be addressed in future studies. Interesting aspects include the influence of the density and/or viscosity ratio on the flow patterns and possible reversal of the flow direction $[8,10]$ as well as symmetry breaking in the azimuthal direction [10]. Moreover, it would be interesting to identify whether similar flow patterns are also observed in mechanically driven oscillating drops with pinned contact lines [3,13] that do not display traveling wave-like surface modes.

$$
* * *
$$

We thank D. VAN DEN ENDE for fruitful discussions and A. STAICU for originally suggesting the Stokes drift mechanism. This work is partially supported by the ExploRe program of "BP Exploration Operating Company Ltd".

\section{REFERENCES}

[1] Rayleigh L., Proc. R. Soc. London A, 29 (1879) 71.

[2] Strani M. and Sabetta F., J. Fluid Mech., 189 (1988) 397.

[3] Basaran O. A. and DePaoli D. W., Phys. Fluids, 6 (1994) 2923.

[4] Noblin X., Buguin A. and Brochard-Wyart F., Eur. Phys. J. E, 14 (2004) 395.

[5] Daniel S., Chaudhury M. K. and De Gennes P.-G., Langmuir, 21 (2005) 4240.

[6] Brunet P., Eggers J. and Deegan R., Phys. Rev. Lett., 99 (2007) 144501.

[7] Mugele F., Baret J.-C. and Steinhauser D., Appl. Phys. Lett., 88 (2006) 204106.

[8] Ko S. H., LeE H. and Kang K. H., Langmuir, 24 (2008) 1094.

[9] Mugele F., Staicu A., Bakker R. and van den Ende D., Lab Chip, 11 (2011) 2011.

[10] Mampallil D., van den Ende D. and Mugele F., Appl. Phys. Lett., 99 (2011) 154102.

[11] Eral H. B., Mampallil Augustine D., Duits M. H. G. and Mugele F., Soft Matter, 7 (2011) 4954.

[12] Dupont J.-B. and Legendre D., J. Comput. Phys., 229 (2010) 2453.

[13] Wilkes E. D. and Basaran O. A., Phys. Fluids, 9 (1997) 1512.

[14] Он J. M., Ko S. H. and KAng K. H., Phys. Fluids, 22 (2010) 032002.

[15] Miller C. A. and Scriven L. E., J. Fluid Mech., 32 (1968) 417.

[16] Basaran O. A., J. Fluid Mech., 241 (1992) 169.

[17] Belonozhko D. F. and Kozin A. V., Fluid Dyn., 46 (2011) 270. 